\title{
High Burden and Depression Among Late-Stage Idiopathic Parkinson Disease and Progressive Supranuclear Palsy Caregivers
}

Journal of Geriatric Psychiatry and Neurology

2017, Vol. 30(5) 267-272

(C) The Author(s) 2017

Reprints and permission:

sagepub.com/journalsPermissions.nav DOI: $10.1177 / 0891988717720300$ journals.sagepub.com/home/jgp

(S)SAGE

\author{
Christian Schmotz, MD ${ }^{1,2}$, Carmen Richinger, BSW ${ }^{1,2}$, and Stefan Lorenzl, MD ${ }^{1,2,3}$
}

\begin{abstract}
Objectives: Caregivers of patients with late-stage idiopathic Parkinson disease (IPD) and late-stage progressive supranuclear palsy (PSP) often suffer from severe psychological strain themselves. This study investigates the influence of the different kind of symptoms in IPD and PSP on the psychological burden of the caregivers. Methods: Twenty patients with late-stage IPD and 20 patients with late-stage PSP and their caregivers were investigated. To measure the degree of motor, cognitive, and affective impairment of the patients, the instruments Subscale III of the Unified Rating Scale for Parkinsonism (UPDRS-III), a shortened 24-item version of the Mini-Mental State Examination, and the Geriatric Depression Scale (GDS-30) were used. Psychological burden of the caregivers was determined by using the Beck Depression Inventory (BDI-II) and the Zarit Caregiver Burden Inventory (ZBI). Results: Patients with IPD suffered from a higher level of depression (GDS-30: I5.9 vs I0.2, $P=.020$ ), whereas patients with PSP showed greater motor impairment (UPDRS-IIl: 38.3 vs 29.9, $P=.002$ ). Caregivers of both groups reported high psychological burden (ZBI: 36.5 in IPD vs 42.8 in PSP) and symptoms of a depression (BDI-II: I 2.5 in IPD vs I5.I in PSP). No significant influence of motor impairment, cognitive dysfunction, and depressive symptoms of the patient on the burden of the caregiver could be found. Conclusions: Psychological strain and depression among caregivers seem to become even more relevant in the late stages of IPD and PSP. Further studies will be necessary to investigate the specific determining factors in late-stage parkinsonian syndromes.
\end{abstract}

\section{Keywords}

burden, caregiver, depression, IPD, late-stage, Parkinson, PSP, progressive supranuclear palsy

\section{Introduction}

Idiopathic Parkinson disease (IPD) represents the most frequent parkinsonian syndrome and the second most frequent neurodegenerative disease in general after Alzheimer disease. Due to the expected change in the future age structure of the population, a further increase in the number of affected people is predicted by experts. ${ }^{1}$ Idiopathic Parkinson disease is a progressive neurodegenerative disease belonging to the group of $\alpha$-synucleinopathies. Whereas many of its core symptoms can be influenced by pharmacological treatment, several nonmotor (eg, dementia, depression, psychosis) and motor problems (eg, postural instability, dysphagia) can occur with further progression of the disease and increasing duration of drug treatment and then show a negative impact on patient's and caregiver's burden as a consequence. ${ }^{2}$ It is clinically very important to distinguish the so-called atypical parkinsonian syndromes progressive supranuclear palsy (PSP), multiple system atrophy (MSA), and corticobasal degeneration (CBD) with their much worse prognosis and much lower response to dopaminergic medication than in IPD. Therefore, palliative care and the alleviation of symptoms get even more important in these diseases. ${ }^{3-5}$
Progressive supranuclear palsy belongs to the group of tauopathies and represents one of the more common variants of atypical parkinsonian syndromes. Progressive supranuclear palsy is a rapidly progressing disease, where patients gradually lose their ability to walk and stand even early in the course of the disease. With further progress, characteristic bulbar symptoms like dysphagia and a frontosubcortical dementia occur frequently. ${ }^{6-8}$

\footnotetext{
' Department of Palliative Medicine, University Hospital, LMU Munich, Munich, Germany

${ }^{2}$ Institute of Nursing Science and Practice, Paracelsus Medical University, Salzburg, Austria

${ }^{3}$ Department of Neurology, Agatharied Hospital, Norbert-Kerkel-Platz, Hausham, Germany
}

Received 2/13/2017. Received revised 5/21/2017. Accepted 6/16/2017.

Corresponding Author:

Christian Schmotz, Department of Palliative Medicine, University Hospital, LMU Munich, Marchioninistrasse 15, 81377 Munich, Germany.

Email: christian.schmotz@med.uni-muenchen.de 
Until now, there is only little data about the circumstances of life of patients with late-stage parkinsonian syndromes-in particular of patients with the atypical variants. The caregivers of patients with IPD are known to suffer from multiple stress factors which are comparable with the ones that can be found in Alzheimer disease. ${ }^{9,10}$ An increasing need of assistance (with dressing, personal hygiene, eating, mobility, application of medication, etc) or general dependence of the patient on the caregiver, growing financial costs, and a lack of support can easily lead to physical and emotional exhaustion of the caregiver-subsumed under the term burden - in the progressive course of the disease. ${ }^{10,11}$ From studies on patients with IPD in earlier stages, it is known so far that the extent of motor and neuropsychiatric symptoms seems to have an important influence on the caregiver's burden, which can reciprocally have an effect on the patient's quality of life and the course of the disease. ${ }^{12,13}$ A similar situation could be assumed for the disabling PSP, but only very little is known about the true burden of the caregivers of patients with PSP. ${ }^{14}$

The aim of this cross-sectional observational study was to compare the psychological strain of caregivers of late-stage patients with IPD and PSP and to scrutinize the influence of the different load of symptoms, as well as the severity of the disease, on the burden of the caregiver.

\section{Methods}

\section{Study Design}

Forty patients diagnosed by a movement disorder specialist (S. Lorenzl, Agatharied; J. Schwarz, Haag) with IPD $(\mathrm{n}=20)$ or PSP $(n=20)$ according to international diagnostic criteria and currently showing a severity of symptoms according to Hoehn and Yahr staging $\geq 4$ and Schwab and England Activities of Daily Living Scale $\leq 50$-representing a late stage of the disease - were included into the study together with their caregivers. As caregivers, we accepted either partners or other family members who had to be involved closely in the all-day care for the patient. Patients with all other kinds of parkinsonian syndromes (eg, MSA, $\mathrm{CBD}$, or drug-induced) as well as earlier stages of IPD and PSP were excluded from our study. Sample size was defined primarily following previous studies and on the basis of the estimated number of patients with PSP available in Southern Germany.

All participants were recruited by contacting self-help groups (German Parkinson Association, German PSP Association), nursing homes, and neurological hospitals with special departments for movement disorders (LMU Munich University Hospital, Agatharied Hospital, Haag Hospital).

First a questionnaire containing the Beck Depression Inventory (BDI-II) was sent to the participants and should be completed by the caregiver on its own. Afterward the participants were visited within 1 week by our research team (physician, social education worker) to conduct the other assessment instruments and to collect the patients' and caregivers' sociodemographic basic data (eg, patient age, disease duration) during an interview. All included patients and caregivers finally participated in the study.
Table I. Limits of MMSE-24.

\begin{tabular}{lcc}
\hline Cognitive impairment & 24-Item Version & 30-Item Version \\
\hline Mild dementia & $16-21$ & $20-26$ \\
Moderate dementia & $8-15$ & $10-19$ \\
Severe dementia & $\leq 7$ & $\leq 9$ \\
\hline
\end{tabular}

Abbreviation: MMSE, Mini-Mental State Examination.

The study had been approved by the local ethics committee of the Ludwig-Maximilians-University of Munich (reference number: 193-14). Written informed consent was obtained from all participants of the study, and none of the participants received any payment or other rewards for taking part.

\section{Instruments}

Data referring to the patient. Besides the Hoehn and Yahr and the Schwab and England Activities of Daily Living Scale, which were used to determine the inclusion criteria, the severity of the illness was assessed by the Unified Rating Scale for Parkinsonism (UPDRS) and the level of motor impairment by the UPDRS-III Subscale. Higher UPDRS scores correspond to a higher severity of symptoms. To evaluate an existing cognitive impairment or dementia, the popular screening tool Mini-Mental State Examination (MMSE) was used. Scores from 0 to 30 points can be achieved, in which lower scores indicate a larger extent of the symptoms. Because of the influence of severe motor dysfunction (as occurring in late-stage parkinsonian syndromes) on the results of the MMSE recognized in previous studies, ${ }^{15}$ we decided to ignore the last 6 items of the test (tasks of writing and drawing), which are strongly connected with the motor performance of the patient, and rather use a shortened 24-item version of the MMSE in order to assess only cognitive dysfunction as far as possible. The limits of the MMSE were adjusted to the 24-item version, as shown in Table 1.

To evaluate the existence of symptoms of depression in the patient, the 30-item version of the Geriatric Depression Scale (GDS-30) was used. Scores from 0 to 30 points can be achieved, in which higher scores indicate a larger extent of the symptoms. Usually, scores from 10 to 19 points are regarded as an indication of mild to moderate and scores from 20 to 30 points as an indication of severe depressive symptoms. In the sense of a categorical evaluation (yes/no), we also assessed anamnestically the existence of any symptoms of psychosis (delusions, hallucinations) at the time of the interview.

Data referring to the caregiver. General psychological strain of the caregivers because of the patient's present situation with the disease was determined by the Zarit Caregiver Burden Inventory (ZBI). It comprises 22 items, which are rated from 0 to 4 points. Higher scores indicate greater burden.

For the assessment of symptoms of depression in the caregivers, the revised version of the BDI-II was used. Scores from 0 to 63 points can be achieved, in which higher scores indicate 
a larger extent of the symptoms. Usually, scores from 9 to 13 points are regarded as minimal, from 14 to 19 points as mild, from 20 to 28 points as moderate, and from 29 to 63 points as severe depressive symptoms.

\section{Data Analysis}

Descriptive statistics were used for sociodemographic and clinical variables. The Kolmogorov-Smirnov test was applied to check for normality of distribution of the data, in order to choose parametric or nonparametric tests for further statistical analyses. Presumed differences in demographic and clinical variables between the groups IPD and PSP were compared using $\chi^{2}$ test in respect of qualitative variables and 2-tailed $t$ test or rather Mann-Whitney $U$ test for quantitative variables. To analyze the relationship between ZBI and BDI-II results, Spearman rank correlation analysis was done. For statistical evaluation of a predictability of ZBI and BDI-II results (dependent variables) by the influence of GDS-30, MMSE-24, UPDRS-III results and the diagnosis of IPD or PSP itself (independent variables), a multiple linear regression analysis was done additionally. Statistical analyses were performed using IBM SPSS Statistics version 23 (IBM Corp., Released 2015, Armonk, NY). A level of significance of $\alpha=.05$ was accepted for all comparisons.

\section{Results}

There were no statistically significant differences in our sample concerning gender of the patients $\left(\chi^{2}=0.100, d f=\right.$ $1, P=.752)$ and gender of the caregivers $\left(\chi^{2}=0.960, d f=1\right.$, $P=.327$ ), which shows the achievement of homogeneity of these characteristics between both groups.

As expected, patients with PSP showed a shorter mean duration of the disease until reaching the late stage compared with patients with IPD. This difference was statistically significant (Mann-Whitney $U$ test: $Z=-4.141, P=.000$ ) and was accompanied by a significantly higher age of the patients in the group of IPD $(t=2.772, d f=38, P=.009)$. Caregivers in both groups did not show a significant difference with regard to age $(t=-0.958, d f=38, P=.344)$.

Symptoms of psychosis seemed to occur more frequently in IPD with a borderline significance $\left(\chi^{2}=3.584, d f=1, P=.058\right)$. One patient with PSP and 1 patient with IPD were not able to communicate at all because of bad condition and therefore could not complete the tasks GDS-30 and MMSE-24. Three patients with PSP were only able to communicate by shaking their head and therefore could not complete the task MMSE-24 but could do the GDS-30. One patient with IPD was not able to understand the questions of GDS-30 due to severe dementia.

As shown in Table 2, further differences between the 2 groups IPD and PSP were seen in the level of symptoms of depression, which ranges from moderate severity in IPD to only very mild in PSP on average. This difference was statistically significant (Mann-Whitney $U$ test: $Z=2.315, P=.020$ ). There were no significant differences with regard to the means of MMSE-24 (Mann-Whitney $U$ test: $Z=-0.551, P=.589$ ) to
Table 2. Sociodemographic and Clinical Variables of Patients and Caregivers in Both Groups of Diagnosis IPD and PSP.

\begin{tabular}{|c|c|c|}
\hline Variables & IPD & PSP \\
\hline Patients & n (\%) & n (\%) \\
\hline Participants & $20(50.0)$ & $20(50.0)$ \\
\hline Men & II (55.0) & $10(50.0)$ \\
\hline Women & $9(45.0)$ & $10(50.0)$ \\
\hline \multirow[t]{2}{*}{ Patients with psychosis } & $7(35.0)$ & $2(10.0)$ \\
\hline & Mean (SD) & Mean (SD) \\
\hline Patient age, years & $76.2(6.4)$ & $70.8(6.0)$ \\
\hline Disease duration, years & I5.3 (7.6) & $6.3(3.0)$ \\
\hline Hoehn and Yahr & $4.3(0.5)$ & $4.9(0.4)$ \\
\hline Schwab and England ADL & $23.5(13.1)$ & $14.0(9.9)$ \\
\hline UPDRS I-IV & $66.5(17.1)$ & $78.7(13.4)$ \\
\hline UPDRS III & $29.9(9.1)$ & $38.3(6.5)$ \\
\hline MMSE-24 ( $N=35 ; \leq 2$ I path) & $18.9(6.2)$ & $18.3(7.1)$ \\
\hline GDS-30 (N $=37 ; \geq 10$ path $)$ & 15.9 (7.2) & $10.2(3.5)$ \\
\hline Caregivers & n (\%) & n (\%) \\
\hline Participants & $20(50.0)$ & $20(50.0)$ \\
\hline Men & $6(30.0)$ & $9(45.0$ \\
\hline \multirow[t]{2}{*}{ Women } & $14(70.0)$ & II (55.0) \\
\hline & Mean (SD) & Mean (SD) \\
\hline Caregiver age, years & $67.1(10.5)$ & $70.0(8.6)$ \\
\hline $\mathrm{ZBI}$ & $36.5(15.3)$ & $42.8(8.7)$ \\
\hline BDI-II [ $\geq 9$ path] & $12.5(5.5)$ & I5.I (8.9) \\
\hline
\end{tabular}

Abbreviations: BDI, Beck Depression Inventory; GDS, Geriatric Depression Scale; IPD, idiopathic Parkinson disease; PSP, progressive supranuclear palsy; SD, standard deviation; UPDRS, Unified Rating Scale for Parkinsonism; ZBI, Zarit Caregiver Burden Inventory.

Table 3. Distribution of MMSE-24 Results in Both Groups.

\begin{tabular}{lcc}
\hline & IPD $(\mathrm{N}=19)$ & PSP $(\mathrm{N}=16)$ \\
\hline No dementia & 9 & 8 \\
Mild dementia & 5 & 5 \\
Moderate dementia & 4 & 1 \\
Severe dementia & 1 & 2 \\
\hline
\end{tabular}

Abbreviations: IPD, idiopathic Parkinson disease; MMSE, Mini-Mental State Examination; PSP, progressive supranuclear palsy.

measure the cognitive impairment between the both groups of patients with IPD and PSP. Both showed a mean cognitive dysfunction in the range of a mild dementia. Detailed results are shown in Table 3. The means of UPDRS-III to measure the motor impairment of the patient differed significantly from IPD to $\operatorname{PSP}(t=3.371, d f=38, P=.002)$.

Caregivers of patients with IPD and PSP reported high to very high mean burden in the ZBI. In BDI-II, their mean scores indicate minimal depression in the group IPD and mild in the group PSP. The differences between both groups were not statistically significant (ZBI: $t=-1.582, d f=30, P=.124$; BDI-II: Mann-Whitney $U$ test: $Z=-0.706, P=.495$ ).

In the whole sample of our study, scores of ZBI as an indicator of general psychological strain of the caregivers and scores of BDI-II as an indicator of symptoms of depression did not show a significant correlation but a trend $(r \mathrm{~s}=.280, P=.081)$. 
Table 4. Regression Analysis for ZBI Scores of Caregivers Within Both Groups.

\begin{tabular}{lcclcc}
\hline IPD & Standardized $\beta$ & $P$ & PSP & Standardized $\beta$ & $P$ \\
\hline UPDRS-III & .173 & .554 & UPDRS-III & -.235 & .480 \\
MMSE-24 & -.209 & .467 & MMSE-24 & -.047 & .885 \\
GDS-30 & .188 & .476 & GDS-30 & .395 & .214 \\
Corrected $R^{2}=-.060, P=.581$ & Corrected $R^{2}=-.070, P=.585$
\end{tabular}

Abbreviations: GDS, Geriatric Depression Scale; IPD, idiopathic Parkinson disease; MMSE, Mini-Mental State Examination; PSP, progressive supranuclear palsy; UPDRS, Unified Rating Scale for Parkinsonism; ZBI, Zarit Caregiver Burden Inventory.

Multiple linear regression analysis revealed that ZBI scores of the caregivers were not determined by the severity of symptoms of the patient with regard to motor impairment (UPDRS-III: standardized $\beta=.136, P=.540$ ), cognitive impairment (MMSE-24: standardized $\beta=-.046, P=$ .807 ), and depressive symptoms (GDS-30: standardized $\beta=$ $.207, P=.286$ ) in our entire sample, whereas the diagnosis of the patient itself showed a statistical trend (standardized $\beta=$ $.374, P=.095)$ to be an independent contributing factor. Age (standardized $\beta=-.170, P=.347$ ) and gender (standardized $\beta=-.047, P=.794$ ) of the caregivers were instead no significant factor. However, there was no statistically significant influence of patient's UPDRS-III, MMSE-24, and GDS-30 on caregiver's ZBI scores (corrected $R^{2}=.065, P=.208$ ). Within the groups IPD and PSP, it was also not possible to predict ZBI scores of the caregivers by the motor, cognitive, or affective severity of symptoms (Table 4).

Furthermore, multiple linear regression analysis showed that BDI-II scores of the caregivers were also not determined by the severity of symptoms of the patient with regard to motor impairment (UPDRS-III: standardized $\beta=.219, P=.341$ ), cognitive impairment (MMSE-24: standardized $\beta=.073$, $P=.705$ ), and depressive symptoms (GDS-30: standardized $\beta=.197, P=.325$ ) in our entire sample. The diagnosis of the patient itself could also not be identified as a contributing factor (standardized $\beta=.236, P=.302$ ), as well as age (standardized $\beta=-.110, P=.547$ ) and gender (standardized $\beta=$ $-.045, P=.802$ ) of the caregivers. However, there was no statistically significant influence of patient's UPDRS-III, MMSE-24, and GDS-30 on caregiver's BDI-II scores (corrected $R^{2}=-.001, P=.426$ ). Within the groups IPD and PSP, it was not possible to predict BDI-II scores of the caregivers by the motor, cognitive, or affective severity of symptoms, although scores seem closer associated in IPD than in PSP (Table 5) and showed a borderline significance $(P=.051)$ for depression in caregivers (BDI-II) and patients (GDS-30) with IPD.

\section{Discussion}

The present study demonstrates that late-stage IPD and latestage PSP usually mean a considerable high psychological burden for the caregivers. In our sample, it was about 1.5 (IPD) to
Table 5. Regression Analysis for BDI-II Scores of Caregivers Within Both Groups.

\begin{tabular}{lcclcc}
\hline IPD & Standardized $\beta$ & $P$ & PSP & Standardized $\beta$ & $P$ \\
\hline UPDRS-III & .334 & .212 & UPDRS-III & .282 & .420 \\
MMSE-24 & .332 & .206 & MMSE-24 & .096 & .778 \\
GDS-30 & .489 & .051 & GDS-30 & -.116 & .721 \\
Corrected $R^{2}=.148, P=.162$ & Corrected & $R^{2}=-.179, P=.866$ \\
\hline
\end{tabular}

Abbreviations: BDI, Beck Depression Inventory; GDS, Geriatric Depression Scale; IPD, idiopathic Parkinson disease; MMSE, Mini-Mental State Examination; UPDRS, Unified Rating Scale for Parkinsonism; PSP, progressive supranuclear palsy.

2 (PSP) times higher than in studies concerning parkinsonian syndromes in earlier stages ${ }^{12,16,17}$ and seem comparable with the results of caregivers of patients with Alzheimer disease. ${ }^{18}$ Caregivers of PSP patients seemed a little more burdened, which could be connected with the rapid progression of the disease and a lack of sufficient options of treatment currently available. Unfortunately, there were not any results available from previous studies about the care of patients with PSP, which emphasizes the necessity of further investigation about the late stages of atypical parkinsonian syndromes.

The extent of reported symptoms of depression (BDI-II) was not correlated statistically significantly with the reported subjective burden in ZBI in our sample but showed a possible connection. Other studies about the burden of caregivers of patients with Alzheimer disease even indicate an independence of reported burden and depression in the caregivers. ${ }^{19}$ This discrepancy may be explained by the influence of individual vulnerability and personality-dependent factors in the pathogenesis of depression ${ }^{20}$ and in addition can often be attributed to an altered view of reality due to intrapsychological resistance of the caregivers confronted with the devastating condition of their beloved one. Altogether, the BDI-II showed that caregivers of both groups of diseases reach scores that indicate the prevalence of a minimal (IPD) to mild (PSP) depression, which is more or less in the range of studies concerning parkinsonian syndromes in earlier stages ${ }^{16,21}$ and shows the persisting need for professional psychological support.

When comparing the symptoms of the diseases IPD and PSP at a similar stage, it gets obvious in our study that patients with PSP suffered from more severe motor impairment, patients of both groups showed mild cognitive dysfunction, whereas patients with IPD seemed to become depressed to a marked extent more easily.

A systematic review showed that in general symptoms of a depression can be found in about $35 \%$ of all patients with IPD and about $17 \%$ fulfill the criteria of a major depressive disorder. $^{22}$ There are only few studies about depression in patients with PSP, mostly carried out in early stages of the disease, which vary considerably in the numbers of prevalence (18\%$60 \%$ ) and the severity of symptoms. ${ }^{21,23-25}$ The noticed lower extent of depression in patients with PSP in our study could be associated with an assumed different pattern of affected brain areas and circuits in the late stage of PSP where orbitofrontal 
and medial frontal circuits are dysfunctional in contrast to IPD where the mesocortical monoaminergic nuclei are involved. ${ }^{26}$

Studies revealed that about $30 \%$ of patients with IPD suffer from dementia and the probability to develop dementia within 10 years after the onset of the disease is about $75 \% .{ }^{27}$ Studies on dementia in patients with PSP detected inconsistent results maybe because of different durations of the disease between the samples. ${ }^{24,28}$ In our study, patients with IPD and PSP showed a mild cognitive impairment probably according to cholinergic dysfunction and loss of white matter found in studies. ${ }^{29,30}$

Besides, patients with PSP showed significantly higher motor impairment than patients with IPD according to the UPDRS-III results. This may be put down to the fact that the caregivers of patients with IPD tried to "present" the patient in a state as good as possible (on-state of the disease) when we visited them, while the caregivers of patients with PSP had no opportunity to influence the state by medication. When taking the on-off state into consideration, our UPDRS-III results seem comparable with other studies. ${ }^{31}$

The prevalence of symptoms of psychosis in our study matches results of previous studies in patients with IPD. ${ }^{32}$ It could be possible that the longer course of the disease IPD, accompanied by a longer treatment with dopaminergic drugs, lead to a higher occurrence of symptoms of psychosis in the late stage of this disease.

In contrast to our results, numerous other studies suggest a connection between motor and neuropsychiatric symptoms of the patients and the burden of the caregivers ${ }^{12,33,34}$ but were done mostly on patients with IPD only. Seeing the (mostly severe) motor and neuropsychiatric symptoms of late-stage patients, having no clear influence on the burden or the occurrence of symptoms of a depression in the caregivers, leads us to the assumption that there are further crucial factors (eg, psychosocial support, financial circumstances, psychological coping with the situation) which need to be investigated in further studies. Furthermore, the borderline significant correlation between depression in patients with IPD and their caregivers, which could not be found within the group of PSP, clearly demonstrates that the different courses of the diseases seem to encourage different ways of intrapsychological processing.

The reduced statistical power due to a small sample size, which is an indirect consequence from the low prevalence of patients with PSP (5 to 10:100 000), can be considered as the major limitation of this study, which other investigations about PSP also faced before. ${ }^{6-8}$ The only way to reach higher statistical power in spite of this rare occurrence of the disease would be the conduction of a multinational study in order to scrutinize the controversial results currently published but exceeded our possibilities.

Further limitations could be seen in the discretionary powers our investigators basically had when applying our instruments and the inclusion criteria. We tried to minimize them by having done all the data assessment by the same investigators to get consistent results. Another limitation might be that caregivers theoretically could have ignored our instructions on fulfilling the questionnaire on their own and instead discussed the answers with the patient or other relatives at first, which could have slightly influenced their finally given statements then. Since the questionnaire did contain instruments affecting the caregiver's very personal feelings of depression and burden, we consider this bias by other persons as very unlikely.

\section{Authors' Note}

The sponsors had no influence on the carrying out of the study or the writing of the manuscript.

\section{Acknowledgments}

The authors thank all patients and their caregivers for taking part, as well as the mentioned self-help groups (German Parkinson Association, German PSP Association), nursing homes, and neurological hospitals (LMU Munich University Hospital, Agatharied Hospital, Haag Hospital) for supporting our study.

\section{Declaration of Conflicting Interests}

The author(s) declared the following potential conflicts of interest with respect to the research, authorship, and/or publication of this article: Stefan Lorenzl has served on the advisory boards for TEVA, Boehringer, and UCB and has received honoraria for scientific presentations from Teva, UCB, and Boehringer.

\section{Funding}

The author(s) disclosed receipt of the following financial support for the research, authorship, and/or publication of this article: This study was partly funded by the German Ministry of Education and Research (BMBF) and the German Parkinson Association (dPV). Stefan Lorenzl has received research support from the German Parkinson Disease Foundation (DPG), German PSP Association (PSP Gesellschaft), German Research Foundation (DFG), and the German Ministry of Education and Research (BMBF).

\section{References}

1. Bach JP, Ziegler U, Deuschl G, Dodel R, Doblhammer-Reiter G. Projected numbers of people with movement disorders in the years 2030 and 2050. Mov Disord. 2011;26(12):2286-2290.

2. Deuschl G, Oertel W, Reichmann H. [DGN S3-Guideline idiopathic Parkinson's disease]. 2016. www.dgn.org. Accessed 2016.

3. Stamelou M, Hoglinger G. A review of treatment options for progressive supranuclear palsy. CNS Drugs. 2016;30(7):629-636.

4. Stamelou M, Bhatia KP. Atypical Parkinsonism: diagnosis and treatment. Neurol Clin. 2015;33(1):39-56.

5. Higginson IJ, Gao W, Saleem TZ, et al. Symptoms and quality of life in late stage Parkinson syndromes: a longitudinal community study of predictive factors. PLoS One. 2012;7(11):e46327.

6. Eggert K, Oertel W, Reichmann H, et al. [Guidelines: Parkinsonian syndromes - diagnostics and therapy]. 2012. www.awmf.org. Accessed 2016.

7. Litvan I, Agid Y, Calne D, et al. Clinical research criteria for the diagnosis of progressive supranuclear palsy (Steele-RichardsonOlszewski syndrome): report of the NINDS-SPSP international workshop. Neurology. 1996;47(1):1-9. 
8. Strzelczyk A, Möller JC, Stamelou M, Matusch A, Oertel WH. Atypical Parkinson syndromes [in German]. Nervenarzt. 2008; 79(10):1203-1220; quiz 1221-1202.

9. Haley WE. The family caregiver's role in Alzheimer's disease. Neurology. 1997;48(5 suppl 6):S25-S29.

10. Hasson F, Kernohan WG, McLaughlin M, et al. An exploration into the palliative and end-of-life experiences of carers of people with Parkinson's disease. Palliat Med. 2010;24(7):731-736.

11. McLaughlin D, Hasson F, Kernohan WG, et al. Living and coping with Parkinson's disease: perceptions of informal carers. Palliat Med. 2011;25(2):177-182.

12. Martinez-Martin P, Rodriguez-Blazquez C, Forjaz MJ, et al. Neuropsychiatric symptoms and caregiver's burden in Parkinson's disease. Parkinsonism Relat Disord. 2015;21(6):629-634.

13. Stella F, Banzato CE, Quagliato EM, Viana MA, Christofoletti G. Psychopathological features in patients with Parkinson's disease and related caregivers' burden. Int J Geriatr Psychiatry. 2009; 24(10):1158-1165.

14. Uttl B, Santacruz P, Litvan I, Grafman J. Caregiving in progressive supranuclear palsy. Neurology. 1998;51(5):1303-1309.

15. Riedel O, Klotsche J, Spottke A, et al. Cognitive impairment in 873 patients with idiopathic Parkinson's disease. Results from the German Study on Epidemiology of Parkinson's Disease with Dementia (GEPAD). J Neurol. 2008;255(2):255-264.

16. Schrag A, Hovris A, Morley D, Quinn N, Jahanshahi M. Caregiver-burden in Parkinson's disease is closely associated with psychiatric symptoms, falls, and disability. Parkinsonism Relat Disord. 2006;12(1):35-41.

17. Grun D, Pieri V, Vaillant M, Diederich NJ. Contributory factors to caregiver burden in Parkinson disease. J Am Med Dir Assoc. 2016;17(7):626-632.

18. Conde-Sala JL, Turró -Garriga O, Calvó -Perxas L, VilaltaFranch J, Lopez-Pousa S, Garre-Olmo J. Three-year trajectories of caregiver burden in Alzheimer's disease. J Alzheimers Dis. 2014;42(2):623-633.

19. Bednarek A, Mojs E, Krawczyk-Wasielewska A, et al. Correlation between depression and burden observed in informal caregivers of people suffering from dementia with time spent on caregiving and dementia severity. Eur Rev Med Pharmacol Sci. 2016;20(1):59-63.

20. Tew EH, Naismith SL, Pereira M, Lewis SJ. Quality of life in Parkinson's disease caregivers: the contribution of personality traits. Biomed Res Int. 2013;2013:151872.
21. Aarsland D, Larsen JP, Karlsen K, Lim NG, Tandberg E. Mental symptoms in Parkinson's disease are important contributors to caregiver distress. Int J Geriatr Psychiatry. 1999;14(10):866-874.

22. Reijnders JS, Ehrt U, Weber WE, Aarsland D, Leentjens AF. A systematic review of prevalence studies of depression in Parkinson's disease. Mov Disord. 2008;23(2):183-189; quiz 313.

23. Schrag A, Sheikh S, Quinn NP, et al. A comparison of depression, anxiety, and health status in patients with progressive supranuclear palsy and multiple system atrophy. Mov Disord. 2010;25(8): 1077-1081.

24. Bloise MC, Berardelli I, Roselli V, et al. Psychiatric disturbances in patients with progressive supranuclear palsy: a case-control study. Parkinsonism Relat Disord. 2014;20(9):965-968.

25. Levy ML, Cummings JL, Fairbanks LA, et al. Apathy is not depression. J Neuropsychiatry Clin Neurosci. 1998;10(3):314-319.

26. Aarsland D, Litvan I, Larsen JP. Neuropsychiatric symptoms of patients with progressive supranuclear palsy and Parkinson's disease. J Neuropsychiatry Clin Neurosci. 2001;13(1):42-49.

27. Aarsland D, Kurz MW. The epidemiology of dementia associated with Parkinson disease. J Neurol Sci. 2010;289(1-2):18-22.

28. Menza MA, Cocchiola J, Golbe LI. Psychiatric symptoms in progressive supranuclear palsy. Psychosomatics. 1995;36(6): 550-554.

29. Roy R, Niccolini F, Pagano G, Politis M. Cholinergic imaging in dementia spectrum disorders. Eur J Nucl Med Mol Imaging. 2016; 43(7):1376-1386

30. Caso F, Agosta F, Volonte MA, et al. Cognitive impairment in progressive supranuclear palsy-Richardson's syndrome is related to white matter damage. Parkinsonism Relat Disord. 2016;31: $65-71$.

31. Kroonenberg PM, Oort FJ, Stebbins GT, Leurgans SE, Cubo E, Goetz CG. Motor function in Parkinson's disease and supranuclear palsy: simultaneous factor analysis of a clinical scale in several populations. BMC Med Res Methodol. 2006;6:26.

32. Bizzarri JV, Giupponi G, Maniscalco I, Schroffenegger P, Conca A, Kapfhammer HP. Parkinson's disease and psychoses [in German]. Neuropsychiatr. 2015;29(1):1-13.

33. Benavides O, Alburquerque D, Chana-Cuevas P. Burden among caregivers of patients with Parkinson disease [in Spanish]. Rev Med Chil. 2013;141(3):320-326.

34. Santos-Garcia D, de la Fuente-Fernandez R. Factors contributing to caregivers' stress and burden in Parkinson's disease. Acta Neurol Scand. 2015;131(4):203-210. 